\title{
Gamma-ray spectral imaging using a single-shutter radiation camera
}

\author{
T.A. DeVol, D.K. Wehe and G.F. Knoll \\ The Universty of Michigan, Ann Arbor, MI 48109-2100, USA
}

\begin{abstract}
As part of a program to develop mobile robots for reactor environments, we are developing a radiation-ımaging camera capable of operatıng in medium-intensity $(<2 \mathrm{R} / \mathrm{h})$, medium-energy $(<8 \mathrm{MeV})$ gamma-ray fields. A systematic study of avalable detectors Indicated the advisability of a high- $Z$ scintillator. The raster-scanning camera uses a lead-shielded bismuth germanate (BGO) scintillator $(1.25 \mathrm{~cm} \times 1.25 \mathrm{~cm}$ right-circular cylinder) coupled to a photomultiplier tube (PMT) operated in pulse mode. Measurements yielded an angular resolution of $2.5^{\circ}$ and energy resolution of $12.9 \%$ at $662 \mathrm{keV}$. The camera motion is totally automated and controlled by stepping motors connected to a remote computer. Several 2D images of radioactive sources have been acquired in fields of up to $400 \mathrm{mR} / \mathrm{h}$ and energies up to $2.75 \mathrm{MeV}$. Some of the images demonstrate the ability of the camera to image a polychromatic field.
\end{abstract}

\section{Introduction}

We are interested in developing a portable radiationImaging camera for imaging gamma-ray fields typically found in a nuclear power station. This portable imager is intended to be mounted on a mobile robot that will take the camera into the radiation area, acquire the image and send the data back to the operator. From a survey of available data on the typical radiation fields in a reactor environment [1], it was determined that under "normal" operating conditions one could expect gamma fields to range up to $8 \mathrm{MeV}$ and $200 \mathrm{R} / \mathrm{h}$. To be practical and useful, it was decided that the camera would be designed for the energy range of 0.5 to $8 \mathrm{MeV}$ and exposures rates up to $2 \mathrm{R} / \mathrm{h}$. There are few applications combining this particular combination of energy range and field strength. Applying the principles from astrophysics and medical imaging cameras, along with physical constraints from our problem, we designed a raster-scanning camera that meets these requirements (fig. 1). The requirement of portability introduced physical constraints on weight, size and efficiency which were important in the design.

\section{Camera design}

The camera consists of a shielded gamma-ray detector mounted on a pan and tilt table. The purpose of the table is to position the camera aperture precisely. Images are formed by recording the count rate of a given ssotope at each orientation of the aperture. By repeatedly moving the aperture and acquiring data, the image is formed pixel by pixel. Since only a single aperture is used, images can be obtained without deconvolution.

\subsection{Detector}

The gamma-ray detector consists of a $1.27 \mathrm{~cm} \times 1.27$ $\mathrm{cm}$ right-circular cylinder of bismuth germanate (BGO) which is coupled to an R1166 photomultiplier tube. BGO was chosen for its high $Z$-value, having an intrinsic peak efficiency that is more than twice that of $\mathrm{NaI}(\mathrm{Tl})$. For a given crystal size, this implies that more full-energy depositions will occur in the BGO than in $\mathrm{NaI}(\mathrm{Tl})$. However, the lower gamma-ray scintillation efficiency of BGO, only $20 \%$ of that of $\mathrm{NaI}(\mathrm{Tl})$, leads to a slightly worse energy resolution than in $\mathrm{NaI}(\mathrm{Tl})$.

Another problem which also leads to decreased energy resolution of $\mathrm{BGO}$ results from its high index of refraction, $n=2.13$. A significant amount of the light created in the crystal will be totally reflected internally when coupled to a glass end-window $(n=1.5)$ PMT. Using Monte Carlo simulations [2] with a $200 \mathrm{~mm}$ bulk absorption mean free path, only about $27 \%$ of the light generated in the scintillator was found to be collected in the PMT. Similar results have been generated elsewhere $[3,4]$. Despite the relatively poor energy resolution of $12.9 \%$ at $0.662 \mathrm{MeV}$, the desire for compactness outweighed the advantage of improved energy resolution for this application, and thus BGO was chosen as the detector element.

\subsection{Mechantcal components}

The radiation detector was centered within the body of the camera. The body was made of ultrapure, low- 

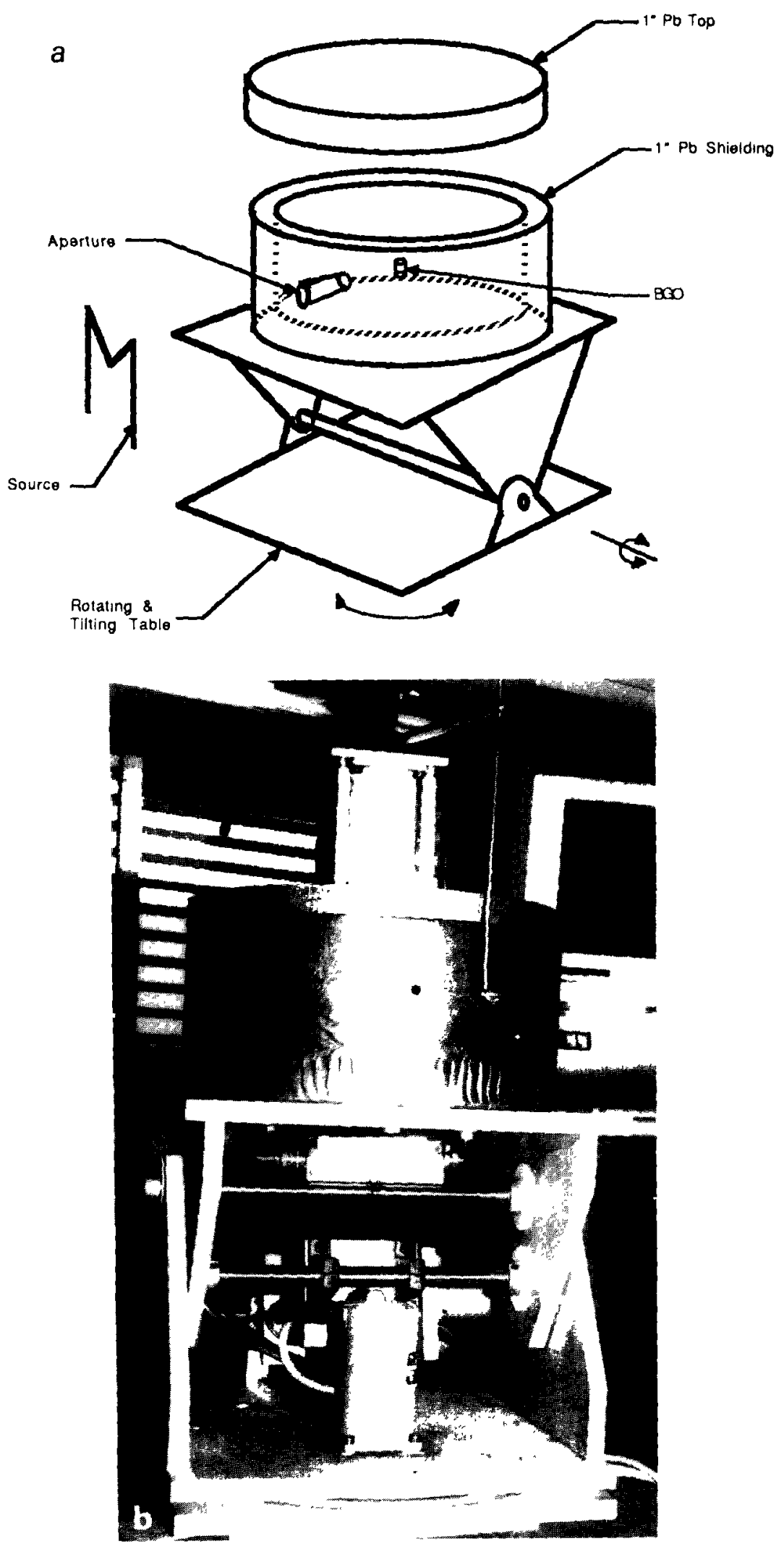

Fig. 1. Schematic (a) and photograph (b) of the raster-scannung camera. 


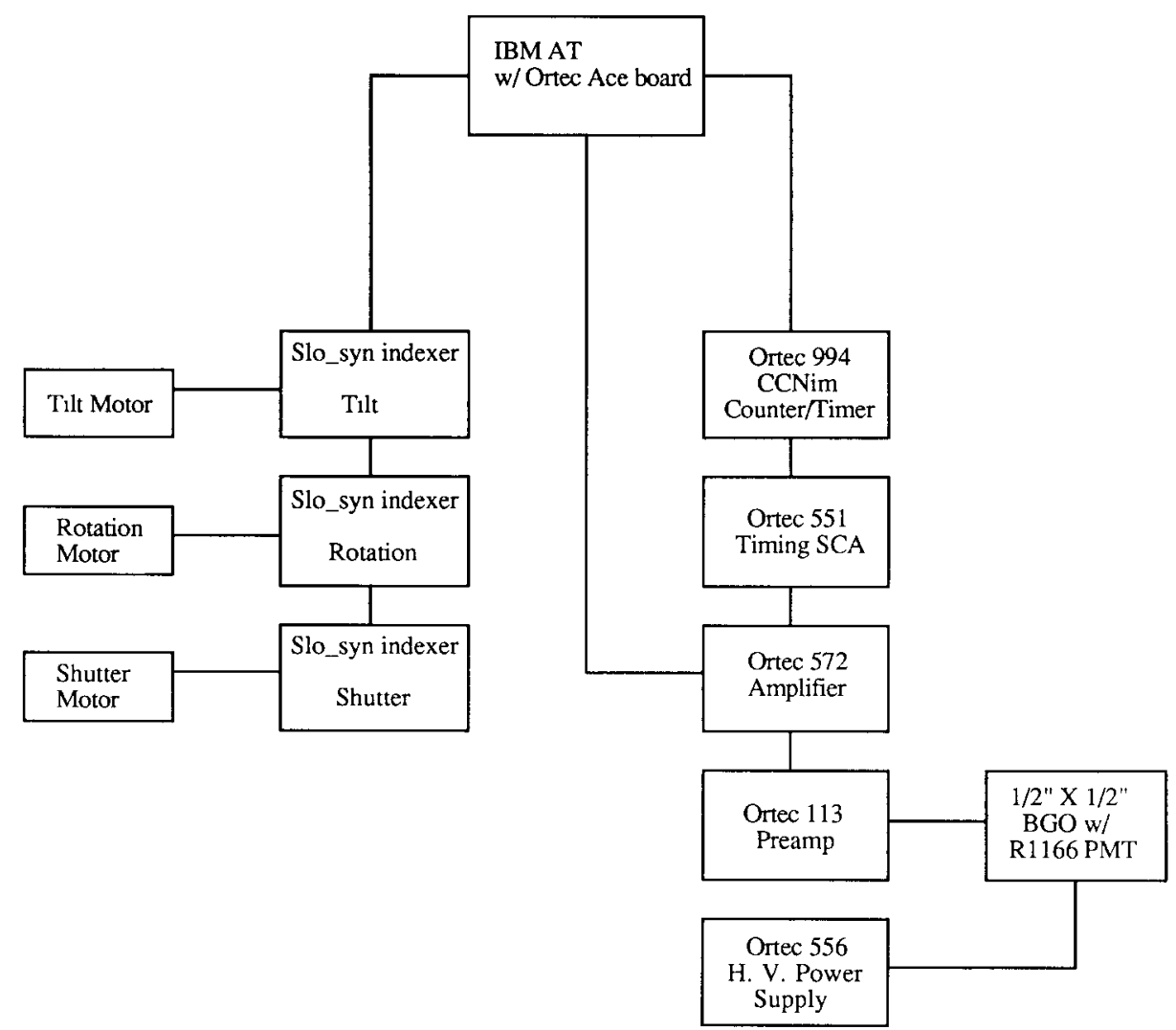

Fig. 2. Schematic of the electronics, showing the interconnections between the computer and the MCA, CCNIM and stepping-motor controllers.

background lead that was formed into a right-circular cylinder having the following dimensions: $27.5 \mathrm{~cm}$ diameter, $15 \mathrm{~cm}$ height and $2.5 \mathrm{~cm}$ thickness. This thickness of lead is sufficient to attenuate incident gamma rays of any energy by at least a factor of 3 . A $0.625 \mathrm{~cm}$ aperture was bored through the shield to determine the incident radiation direction. However, since the gammaray background penetrating through the camera body will usually exceed the flux passing through the aperture, a mechanical shutter was used to improve the image contrast. Contrast, defined as the ratio of the count rates of a "hot" pixel divided by those of a "cold" pixel, is used as a figure of merit to determine the ability to seperate points from the background. The shutter consists of a lead plug which simulates a closed aperture. The shutter is mechanically moved using an automated stepping motor.

Subtracting the count rate acquired with the aperture closed from the count rate acquired with the aperture open yields a difference that is a measure of the gamma rays which passed through the open aperture. The technique was highly effective in improving image contrast.

The positioning of the camera is performed by the underlying table. The table has two degrees of freedom, allowing to tilt $\pm 20^{\circ}$ about a horizontal axis and rotate $\pm 180^{\circ}$ about a vertical axis. The computer-controlled dc stepping motors (cf. fig. 2) enabled the aperture to be positioned to within $1^{\circ}$. The motors' steps are driven through a command to rotate a given number of motor steps, translating directly into a camera motion of a known angular deflection. For our experiments, camera movement was taken in $0.54^{\circ}$ or $1.04^{\circ}$ steps. The table

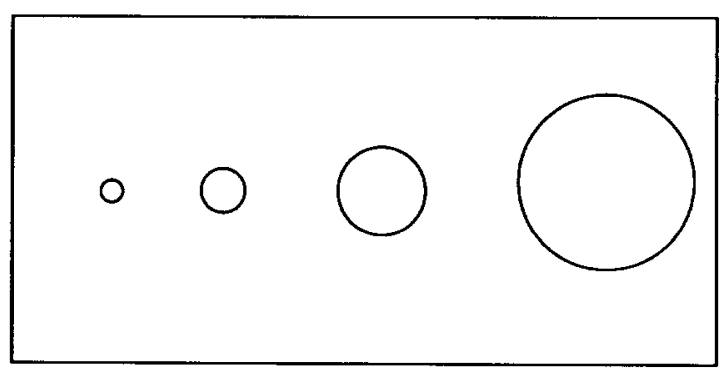

F1g. 3 Radioactive $(\sim 150 \mu \mathrm{Cu}){ }^{95} \mathrm{Zr}$ plate with dimensions of $5 \mathrm{~cm} \times 10 \mathrm{~cm}$. Hole diameters from left to right: $0.318,0.625$, 1.27 and $2.54 \mathrm{~cm}$. 

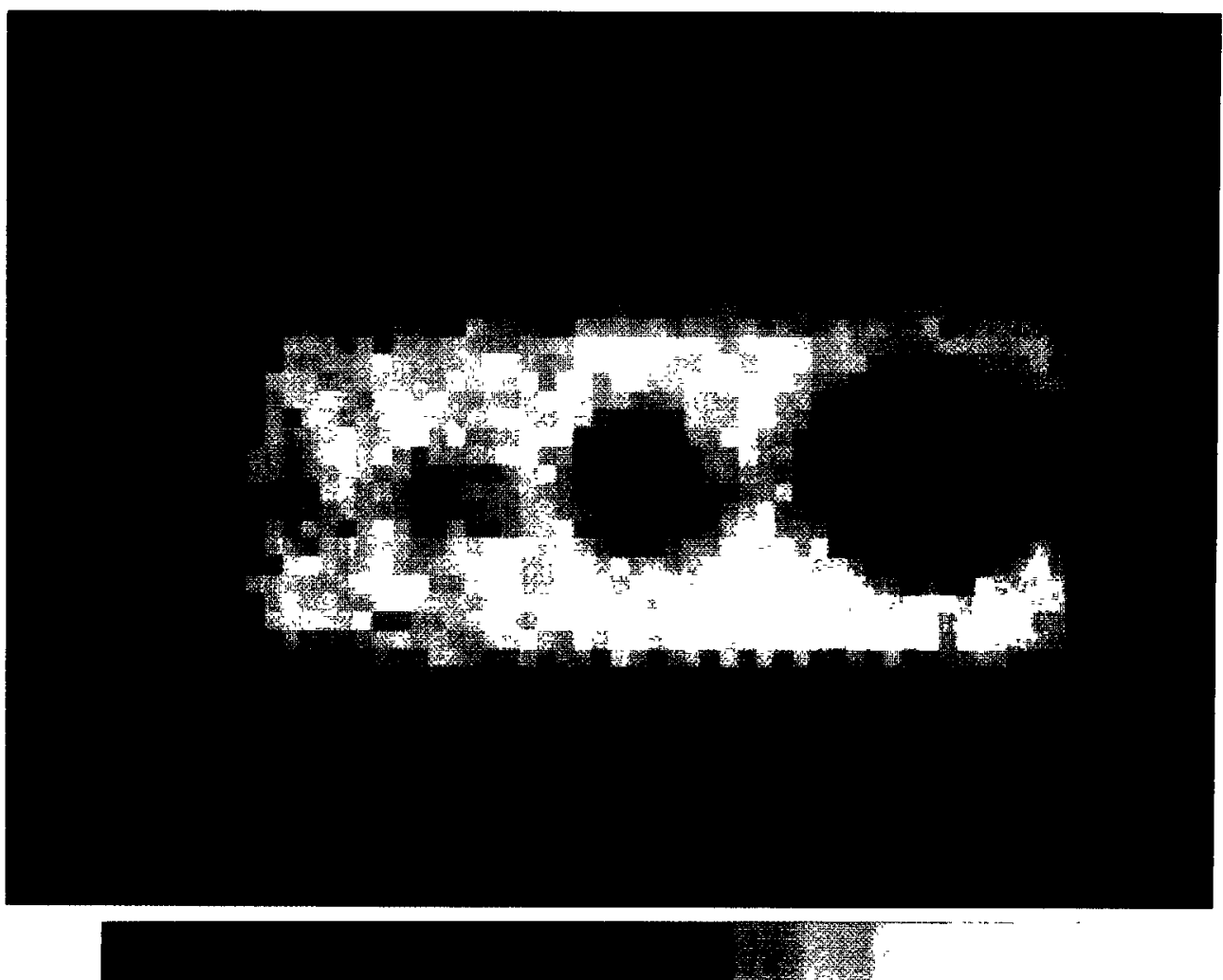

Fig 4. Radiation image of the 0724 and $0756 \mathrm{MeV}$ gamma ravs of ${ }^{\circ \times} \mathrm{Zr}$ The pixel dimension are $0159 \mathrm{~cm} \times 0159 \mathrm{~cm}$

has demonstrated excellent position reproducibility. to withın $0.07^{\circ}$, while supporting $200 \mathrm{lbs}$

\subsection{Electronlcs}

The pulse-processing electronics associated with the camera are shown in fig. 2 and consist of a preamplifier (ORTEC 113), an amplifier (ORTEC 572) and an SCA (ORTEC 551). The SCA was used to bracket the isotope of interest and permitted selective spectral ımagIng. The SCA was connected to a computer-controlled counter and tımer (ORTEC 994). Data were acquired at each pixel using the C NIM to determine the net count rate of each isotope of interest. The camera position and net count rates were stored on a computer disk for later image display

\section{Image displar}

The image data were acquired on an IBM AT computer These data files were transferred to Apollo workstations for processing and display. Since the camera has good spatial resolution. processing simply involved extractıng the net count rate and forming a normalized

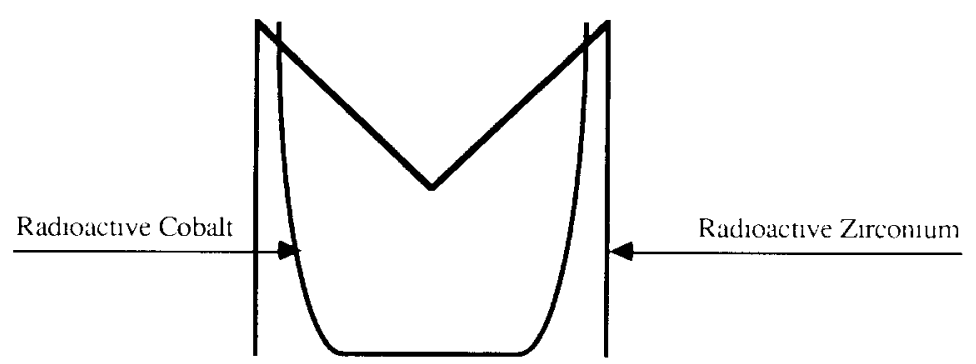

Fig. 5. Schematic representation of two radioactive sources The "U" is " $\mathrm{C}$ ( $\mathrm{U}$ and the " $\mathrm{M}$ " ${ }^{14}$ " $\mathrm{Zr}$ Both sources occupv an area of 10 $\mathrm{cm} \times 10 \mathrm{~cm}$ and are $-100 \mu \mathrm{C}$ ' each 

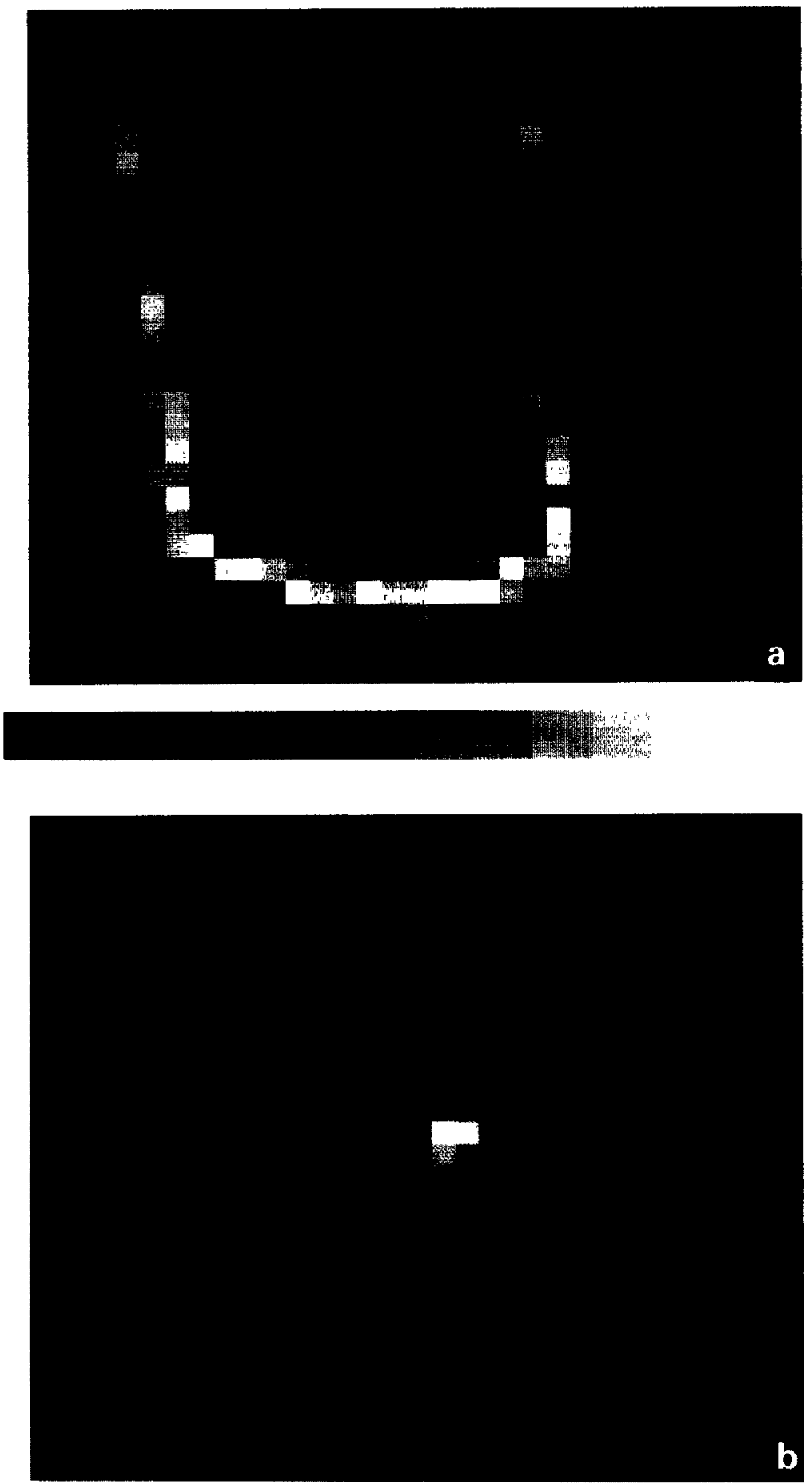

Fig. 6. (a) Radiation image of the 1.173 and $1.332 \mathrm{MeV}$ gamma rays of ${ }^{60} \mathrm{Co}$. (b) Radiation image of the 0.724 and $0.756 \mathrm{MeV}$ gamma rays of ${ }^{95} \mathrm{Zr}$. The pixel dimensions for both images are $0318 \mathrm{~cm} \times 0.318 \mathrm{~cm}$. 
image file. Since the images shown here had a known incident energy, color coding was used to visually display the intensity. The color palette chosen for display is a black-body radiator, i.e., white is the most intense and deep red the least intense.

\section{Experimental results}

The current camera design went through several revisions, primarily in the shuttering mechanism, to improve 1ts point-spread function. It was discovered experimentally and by Monte Carlo simulation that the signal-to-noise ratio and point-spread function are extremely sensitive to exact alignment of the shutter and the aperture. By simulating a closed aperture with a 2.5 $\mathrm{cm}$ long $\times 0.635 \mathrm{~cm}$ diameter lead cylinder that just covered the aperture, an angular resolution of $\sim 2.5^{\circ}$ and a contrast of $\sim 8$ were achieved. This resulted in a point-spread function which produced images that could be displayed without deconvolution.

High-purity metals were irradiated in the Ford Nuclear Reactor at the University of Michigan to produce the radiation sources used to test the camera. These radioactive sources ranged in energy from 0.724 to $2.75 \mathrm{MeV}$ and had activities up to $400 \mu \mathrm{Ci}$. The sources were shaped into various geometries and positioned about $25 \mathrm{~cm}$ in front of the detector. The test source shown in fig. 3 was used to characterize the spatial resolution of the camera. The source is a solid radioactive $(150 \mu \mathrm{Ci}){ }^{95} \mathrm{Zr}$ plate with four holes punched out with diameters of $2.5,1.27,0.635$ and $0.318 \mathrm{~cm}$. The source produced a field of less than $10 \mathrm{mR} / \mathrm{h}$ at the camera and resulted in a typical net count rate of 32 cps. The resulting image is shown in fig. 4 . Note that the 2.54 and $1.27 \mathrm{~cm}$ diameter holes are clearly recognizable, and the $0.635 \mathrm{~cm}$ hole is the limit at which we can resolve objects spatially. This image was produced by quarter-stepping the camera motion so that four count-rate measurements were taken for each field of view. In terms of the image, each pixel represents a physical dimension of $0.159 \mathrm{~cm} \times 0.159 \mathrm{~cm}$. This is consistent with a $5 \mathrm{~cm} \times 10 \mathrm{~cm}$ plate and the hole dimensions cited above.

Fig. 5 shows another example which tests spatial resolution as well as spectroscopic ability. Two radioactive sources were used: a ${ }^{60} \mathrm{Co}$ wire formed into a " $U$ " and a zirconium strip formed into an " $M$ ". Each source was $-100 \mu \mathrm{Ci}$, which yielded a net count rate of $3 \mathrm{cps}$ for the cobalt (fig. 6a) and $36 \mathrm{cps}$ for the zirconıum (fig. $6 \mathrm{~b}$ ). These images were produced by half-stepping the camera motion, which translates into a pixel area of $0.318 \mathrm{~cm} \times 0.318 \mathrm{~cm}$. Both sources occupied an area of approximately $10 \mathrm{~cm} \times 10 \mathrm{~cm}$. The asymmetry in the images reflects the true shapes of the sources. Note that the intense region in fig. $6 \mathrm{~b}$ is real and is caused by increased activity due to overlapping folds of metal in that region. This observation is consistent with the intensity difference in the image.

\section{Future efforts}

The current version of our gamma-ray camera has yielded good spatial resolution and is able to perform spectroscopic imaging. The low $\left(1.06 \times 10^{-6}\right)$ absolute detection effiency for a full-energy deposition of a 1 $\mathrm{MeV}$ gamma ray withn the field of view at $1 \mathrm{~m}$ permits imaging in relatively intense fields in a reasonable amount of time. However, we are currently in the process of reconfiguring our camera to increase this efficiency in order to be able to image lower-intensity fields. The current camera has imaged fields up to 15 $\mathrm{mR} / \mathrm{h}$, but requires inordinately long periods to collect images of low-intensity fields with good statistics. With a more efficient camera, we will acquire images of these lower-intensity fields considerably faster.

\section{Acknowledgements}

We would like to thank Jim Ottaviani for his assessment of typical reactor radiation fields and his assistance with data acquisition using the early prototype cameras, Nesrın Dogan for her work in characterızıng the camera response using Monte Carlo methods, Yuval Roth-Tabak for his help writing the image display program, and Jim McCartney, Yechial Weinstein and Yigal Shaul for their help in the mechanical design of the table.

\section{References}

[1] J. Ottaviani and D.K. Wehe, Ann. Nucl. Energy 16 (1989) 91.

[2] G F. Knoll, T.F. Knoll and T.M. Henderson, IEEE Trans. Nucl. Sc1. NS-35 (1988) 872.

[3] S.E. Derenzo and J.K. Riles, IEEE Trans Nucl. Scı. NS-29 (1982) 191

[4] S.E. Derenzo, T F. Budinger and T. Vuletich, IEEE Trans Nucl. Sc1, NS-30 (1983) 665. 